[Article]

www.whxb.pku.edu.cn

\title{
氧化铝载体和氧化钡助剂对钌基氨合成催化剂结构和性能的影响
}

\author{
杨晓龙 ${ }^{1,2}$ 夏春谷 ${ }^{1}$ 唐立平 ${ }^{1,2}$ 熊绪茂 ${ }^{1}$ 慕新元 ${ }^{1}$ 胡 斌 ${ }^{1, *}$ \\ ('中国科学院兰州化学物理研究所, 兰州 730000; 2中国科学院研究生院, 北京 100049)
}

\begin{abstract}
摘要: 采用不同来源 $\gamma-\mathrm{Al}_{2} \mathrm{O}_{3}$ (市售 $\mathrm{Al}_{2} \mathrm{O}_{3}-1$, 合成 $\mathrm{Al}_{2} \mathrm{O}_{3}-2$ ) 作为钓基氨合成催化剂载体, 利用浸渍法制备了一系 列添加不同 $\mathrm{BaO}$ 助剂含量的 $\mathrm{Ba}-\mathrm{Ru} / \mathrm{Al}_{2} \mathrm{O}_{3}$ 催化剂. 通过 $X$ 射线衍射 (XRD)、 $\mathrm{N}_{2}$-低温物理吸附、X射线苂光光谱 $(X R F) 、$ 透射电镜 $(T E M) 、 H_{2}$ 程序升温还原 $\left(\mathrm{H}_{2}-\mathrm{TPR}\right) 、 \mathrm{NH}_{3}$ 程序升温脱附 $\left(\mathrm{NH}_{3}-\mathrm{TPD}\right)$ 和 $X$ 射线光电子能谱 $(X P S)$ 等 方法研究了不同来源的 $\mathrm{Al}_{2} \mathrm{O}_{3}$ 以及 $\mathrm{BaO}$ 助剂含量对负载型钓基催化剂的物相结构、织构性质、微观形貌、表面性 质和催化剂的氨合成活性等方面的影响. 结果表明, 载体的物理化学性质对制备的钓基氨合成催化剂的结构以 及活性有较大影响. $\mathrm{BaO}$ 助剂对催化剂的影响主要表现在两个方面: 添加量不同导致 $\mathrm{BaO}$ 与 $\gamma-\mathrm{Al}_{2} \mathrm{O}_{3}$ 的作用力 不同, 从而进一步影响催化体系的比表面积和孔结构性质; $\mathrm{BaO}$ 助剂会对体系的 Ru物种还原性质以及催化剂 表面酸碱性质进行调节, 适量 $\mathrm{BaO}$ 的加入能够极大提高反应活性, 而这种最佳量与载体性质密切相关.
\end{abstract}

关键词: 氨合成; $\gamma-\mathrm{Al}_{2} \mathrm{O}_{3} ;$ 载体; $\mathrm{Ru}$; 还原; 酸性 中图分类号: $0643.3 ; \mathrm{TQ} 113.2$

\section{Effect of Alumina Support and Barium Oxide on the Structure and Catalytic Activity of Ruthenium Catalysts for Ammonia Synthesis}

\author{
YANG Xiao-Long ${ }^{1,2} \quad$ XIA Chun-Gu $\quad$ TANG Li-Ping ${ }^{1,2} \quad$ XIONG Xu-Mao' \\ MU Xin-Yuan ${ }^{1} \quad$ HU Bin ${ }^{1, *}$ \\ ('Lanzhou Institute of Chemical Physics, Chinese Academy of Sciences, Lanzhou 730000, P. R. China; \\ ${ }^{2}$ Graduate University of Chinese Academy of Sciences, Beijing 100049, P. R. China)
}

\begin{abstract}
A series of $\mathrm{Ba}-\mathrm{Ru} / \mathrm{Al}_{2} \mathrm{O}_{3}$ catalysts were prepared by the impregnation method using industrial alumina $\left(\mathrm{Al}_{2} \mathrm{O}_{3}-1\right)$ and synthesized alumina $\left(\mathrm{Al}_{2} \mathrm{O}_{3}-2\right)$ as supports. The catalysts were characterized by $\mathrm{X}$-ray diffraction, $\mathrm{N}_{2}$ adsorption-desorption, $\mathrm{X}$-ray fluorescence spectroscopy, transmission electron microscopy, $\mathrm{H}_{2}$ temperature-programmed reduction, $\mathrm{NH}_{3}$ temperature-programmed desorption, and $\mathrm{X}$-ray photoelectron spectroscopy. The effect of $\mathrm{Al}_{2} \mathrm{O}_{3}$ and the $\mathrm{BaO}$ promoter on the phase structure, texture properties, morphology, surface properties, and catalytic activity in ammonia synthesis were investigated. The results indicate that the physical and chemical properties of $\mathrm{Al}_{2} \mathrm{O}_{3}$ have a strong impact on the structure and activity of the ruthenium catalysts. The $\mathrm{BaO}$ promoter has a strong impact on the ruthenium catalyst in two ways: first, the amount of $\mathrm{BaO}$ added leads to a difference in the interaction between $\mathrm{BaO}$ and $y-\mathrm{Al}_{2} \mathrm{O}_{3}$, which further influences the specific area and the porous structure of the catalysts; second, the addition of $\mathrm{BaO}$ influences the reduction process and the surface acidity and alkaline properties of the ruthenium catalysts. A proper amount of $\mathrm{BaO}$ promotes the activity and the optimal amount of $\mathrm{BaO}$ depends on the properties of the supports.
\end{abstract}

Key Words: Ammonia synthesis; $\quad \mathrm{V}-\mathrm{Al}_{2} \mathrm{O}_{3} ;$ Support; Ruthenium; Reduce; Acidity

Received: September 1, 2010; Revised: September 29, 2010; Published on Web: November 8, 2010.

"Corresponding author. Email: hcom@lzb.ac.cn; Tel: +86-931-4968258.

The project was supported by the National Science Foundation for Distinguished Young Scholars of China (20625308).

国家杰出青年科学基金(20625308)资助项目

(C) Editorial office of Acta Physico-Chimica Sinica 
钉基氨合成催化剂被誉为新一代氨合成催化 剂, 其研究与开发成为当今国内外学者关注的热点 之一 ${ }^{[1-10]}$. 与铁基催化剂相比, 钓基催化剂的主要优 点是低温低压活性高, 对水、 $\mathrm{CO}$ 和 $\mathrm{CO}_{2}$ 不敏感, 受 $\mathrm{NH}_{3}$ 的抑制作用不明显, 可在较高的氨浓度下操作, 因此是理想的低温低压氨合成催化剂 ${ }^{[2]}$. 英国 BP 公 司于 1979 年开发了石墨化活性炭负载钉基氨合成 催化剂, 之后, BP 公司和美国 Kellogg 公司合作, 于 1990 年成功开发出全球第一套以石墨化活性炭负 载钓基氨合成催化剂为基础的 KAAP(Kellogg Advanced Ammonia Process)工艺流程. 投产后的运行 情况表明钓基催化剂的寿命与活性炭载体的流失 密切相关, 在氨合成反应条件下, $\mathrm{Ru}$ 能催化活性炭 甲烷化反应. 因此, 活性炭载体的稳定性及其在工 业使用过程中的流失是一个重要的问题. 此外, 活 性炭载体的石墨化过程条件苛刻、生产成本较高、 操作与控制比较复杂 ${ }^{[2,4]}$, 这些因素极大地阻碍了钉 基催化剂的工业化应用进程.

针对这些问题, 寻找优良载体成为新一代钓基 氨合成催化剂研究开发的关键, 载体应在氨合成条 件下具有较高的稳定性, 一定的机械强度, 合适的 比表面积和孔结构. 目前, 一些难还原的金属氧化 物, 如 $\mathrm{Al}_{2} \mathrm{O}_{3}{ }^{[4,9-11]} 、 \mathrm{MgO}^{[6,8,12]} 、 \mathrm{MgAl}_{2} \mathrm{O}_{4}{ }^{[5]}$ 等已被广泛研 究. 通过对金属氧化物载体负载钉基氨合成催化剂 的系统研究, Aika 等 ${ }^{[3]}$ 认为载体的电负性越小(即碱 性越强), 催化剂的活性越高. $\mathrm{Al}_{2} \mathrm{O}_{3}$ 作为一种最常用 的载体, 由于表面存在较多的 Lewis 酸性中心, 因此 氨合成活性相对较低, 被普遍认为不适于做钉基催 化剂载体. 张新波等 ${ }^{[4]}$ 认为, $\mathrm{Al}_{2} \mathrm{O}_{3}$ 具有比表面积较 高、化学性质稳定和机械强度较好等优点, 可以通 过修饰或改变其表面性质(如降低其表面酸性, 提高 表面导电性)等方法, 提高 $\mathrm{Al}_{2} \mathrm{O}_{3}$ 负载钉基催化剂的 氨合成活性. 虽然, $\mathrm{Al}_{2} \mathrm{O}_{3}$ 具有成为工业化钓基氨合 成催化剂载体的潜力, 然而, 目前国内对 $\mathrm{Al}_{2} \mathrm{O}_{3}$ 负载 钉基催化剂的详细研究报道还不多 ${ }^{[4,13-14]}$. 我们从两 个方面对 $\mathrm{Al}_{2} \mathrm{O}_{3}$ 负载钉基催化剂开展系统研究: $\gamma-\mathrm{Al}_{2} \mathrm{O}_{3}$ 载体和 $\mathrm{BaO}$ 助剂对钓基催化剂结构和氨合 成活性的影响. 探索不同来源 $\mathrm{Al}_{2} \mathrm{O}_{3}$ 作为钉基氨合成 催化剂载体的异同点, $\mathrm{BaO}$ 助剂的促进作用如何受 载体的影响等, 探讨 $\mathrm{Al}_{2} \mathrm{O}_{3}$ 载体和 $\mathrm{BaO}$ 助剂影响催 化剂性质和氨合成活性的本质原因.

\section{1 载体的合成}

工业用氧化铝 $(\gamma$ 型, GA-385, 江苏省三剂实业 有限公司)在 $500^{\circ} \mathrm{C}$ 马弗炉中焙烧 $6 \mathrm{~h}$, 研磨备用, 记 为 $\mathrm{Al}_{2} \mathrm{O}_{3}-1$. 将一定量的 $\mathrm{Al}\left(\mathrm{NO}_{3}\right)_{3} \cdot 9 \mathrm{H}_{2} \mathrm{O}$ (分析纯, 北京 化工厂) 溶解在蒸馏水中, 室温下配制成 $0.2 \mathrm{~mol} \cdot \mathrm{L}^{-1}$ 的水溶液, 搅拌条件 $\left(700 \mathrm{r} \cdot \mathrm{min}^{-1}\right)$ 下滴加 $0.6 \mathrm{~mol} \cdot \mathrm{L}^{-1}$ 的 $\mathrm{Na}_{2} \mathrm{CO}_{3}$ (分析纯, 国药集团化学试剂有限公司)水 溶液, 待成胶完全后将反应液静置过夜、抽滤、洗 涤, 所得白色沉淀 $110^{\circ} \mathrm{C}$ 干燥 $12 \mathrm{~h}$, 在马弗炉中 $500{ }^{\circ} \mathrm{C}$ 焙烧 $6 \mathrm{~h}$, 研磨备用, 记为 $\mathrm{Al}_{2} \mathrm{O}_{3}-2$.

$\mathrm{Al}_{2} \mathrm{O}_{3}$ 载体的组成分析结果列于表 1 .

\section{2 钉基氨合成催化剂的制备}

采用浸渍法制备钉基氨合成催化剂, $\mathrm{Ru}$ 负载的 质量分数为 $5 \%$. 将载体用 $\mathrm{Ru}_{3}(\mathrm{CO})_{12}$ (自制)的四氢 呋喃 (THF) (分析纯, 天津化学试剂有限公司)溶液 浸渍 $12 \mathrm{~h}$, 室温蒸去溶剂, 于 $60^{\circ} \mathrm{C}$ 真空干燥 $12 \mathrm{~h}$, 再 在 $300{ }^{\circ} \mathrm{C}$ 下 $\mathrm{H}_{2}$ 气氛加热 $3 \mathrm{~h}$ 以分解 $\mathrm{Ru}_{3}(\mathrm{CO})_{12}$. 所得 样品分为两部分: 一部分经压片、破碎、笁分后得无 助剂的钓基催化剂 $\mathrm{Ru} / \mathrm{Al}_{2} \mathrm{O}_{3}$; 另一部分用 $\mathrm{Ba}\left(\mathrm{NO}_{3}\right)_{2}$ (分析纯, 上海化学工业专科学校实验工厂) 的水溶 液浸渍 $12 \mathrm{~h}, 110^{\circ} \mathrm{C}$ 干燥 $12 \mathrm{~h}$, 压片、破碎、篮分制备 $\mathrm{Ba}: \mathrm{Ru}$ 摩尔比分别为 $1: 1 、 3: 1 、 5: 1$ 的 $\mathrm{Ba}-\mathrm{Ru} / \mathrm{Al}_{2} \mathrm{O}_{3}$ 催 化剂.

\section{3 催化剂的氨合成活性评价}

氨合成反应在固定床反应器中进行, 催化剂 (20-40 目)用量 $2 \mathrm{~mL}$, 反应压力 $3.0 \mathrm{MPa}$, 反应气为 $\mathrm{H}_{2}$ 和 $\mathrm{N}_{2}$ 混合气, 空速 $5000 \mathrm{~h}^{-1}$. 活性测试前催化剂在 常压下用反应气在 $450^{\circ} \mathrm{C}$ 活化 $6 \mathrm{~h}$, 然后在测试条件 下至少稳定 $2 \mathrm{~h}$, 产物氨由气相色谱 (Shimadzu GC-9A PORAPAK QS)在线分析. 催化剂活性 $(x)$ 由 下式计算得到的单位时间内单位质量催化剂上生 成的氨体积量 $\left(\mathrm{mL} \cdot \mathrm{g}^{-1} \cdot \mathrm{h}^{-1}\right)$ 表示.

$$
\begin{aligned}
x & =\left[\varphi\left(\mathrm{NH}_{3}\right) \times \mathrm{GHSV} \times V_{\text {cat }}\right] / m_{\text {cat }} \\
& =\left[\varphi\left(\mathrm{NH}_{3}\right) \times 5000 \times 2\right] / m_{\text {cat }}
\end{aligned}
$$

其中: $m_{\mathrm{cat}}$ 是催化剂质量, 单位 $\mathrm{g}$; GHSV (gas hourly space velocity) 是体积空速, 单位 $\mathrm{h}^{-1} ; V_{\text {cat }}$ 是催化剂体

表 $1 \mathrm{Al}_{2} \mathrm{O}_{3}$ 的组成 $(\%)$ 分析

Table 1 Composite (\%) analyses of $\mathrm{Al}_{2} \mathrm{O}_{3}$ samples

\begin{tabular}{cccccc}
\hline Sample & $w\left(\mathrm{Al}_{2} \mathrm{O}_{3}\right)$ & $w\left(\mathrm{SiO}_{2}\right)$ & $w\left(\mathrm{Na}_{2} \mathrm{O}\right)$ & $w(\mathrm{MgO})$ & $w(\mathrm{CaO})$ \\
\hline $\mathrm{Al}_{2} \mathrm{O}_{3}-1$ & 99.14 & 0.35 & 0.14 & $<0.01$ & 0.19 \\
$\mathrm{Al}_{2} \mathrm{O}_{3}-2$ & 93.90 & 3.03 & 2.57 & 0.16 & $<0.01$ \\
\hline
\end{tabular}

$\mathrm{Al}_{2} \mathrm{O}_{3}-1$ : commercial $\gamma$-alumina; $\mathrm{Al}_{2} \mathrm{O}_{3}-2$ : $\gamma$-alumina prepared by precipitation method

\section{1 实 验}


积, 单位 $\mathrm{mL}$.

\section{4 催化剂的表征}

XRF 在荷兰 PANalytical 公司的 Magix PW2403 $X$ 射线荧光光谱仪上进行. XRD 测试在荷兰 PANalytical 公司 X'pert PRO 型XRD 衍射仪上进行, 采用 $\mathrm{Cu} K_{a}$ 射线, 管电压 $40 \mathrm{kV}$, 管电流 $40 \mathrm{~mA}, 2 \theta$ 扫描范 围为 $15^{\circ}-85^{\circ}$, 扫描速率 $0.2\left(^{\circ}\right) \cdot \mathrm{s}^{-1}$. $\mathrm{N}_{2}$ - 低温物理吸 附在 ASAP 2010 型气体吸附仪(USA Micromeritics) 上于 $-196{ }^{\circ} \mathrm{C}$ 进行, 测试前, 样品在 $200^{\circ} \mathrm{C}$ 真空条件 下脱气处理 $5 \mathrm{~h}$. 样品的比表面积采用 BET 方程计 算求得; 孔容取 $p / p_{0}=0.99$ 时相应的吸附体积; 孔径 分布曲线由 $\mathrm{BJH}$ 公式计算得到. 催化剂微观形貌由 日本 JEOL 公司的 JEM-2010型透射电子显微镜得 到. 测试前样品加入乙醇溶液中用超声波分散, 在 微栅上晾干后直接拍摄, 电子加速电压 $200 \mathrm{kV}$, 分 辨率为 $0.194 \mathrm{~nm}$. $\mathrm{H}_{2}$-TPR 测试采用先权公司 TP-5080 型全自动多用吸附仪. 具体操作为: 称取 $50 \mathrm{mg}$ 催化剂样品置于石英管中, $200{ }^{\circ} \mathrm{C}$ 下高纯 $\mathrm{Ar}$ 吹扫 $30 \mathrm{~min}$, 降至室温, 继续用高纯 $\mathrm{Ar}$ 吹扫至基线 平稳. 以 $10 \% \mathrm{H}_{2}-90 \% \mathrm{Ar}$ 混合气进行程序升温还原 至 $700{ }^{\circ} \mathrm{C}$, 还原气流速 $30 \mathrm{~mL} \cdot \mathrm{min}^{-1}$, 升温速率 $10^{\circ} \mathrm{C}$. $\mathrm{min}^{-1}$. 利用 $\mathrm{NH}_{3}$-TPD 化学吸附法测定样品酸碱性. 采用先权公司 TP-5080 型全自动多用吸附仪. 具体 操作为: 称取 $100 \mathrm{mg}$ 催化剂样品于石英管中, 在流 速为 $20 \mathrm{~mL} \cdot \mathrm{min}^{-1}$ 的高纯 $\mathrm{Ar}$ 气流中升温至 $450{ }^{\circ} \mathrm{C}$, 吹扫样品 $1 \mathrm{~h}$ 以脱除吸附在样品表面的杂质, 然后 在 $\mathrm{Ar}$ 气流中降至室温, 将 $\mathrm{Ar}$ 气流切换成高纯 $\mathrm{NH}_{3}$ 气流, 室温下吸附 $\mathrm{NH}_{3} 1 \mathrm{~h}$, 吸附完毕后切换回流速 为 $20 \mathrm{~mL} \cdot \mathrm{min}^{-1}$ 的 $\mathrm{Ar}$ 气流, 于 $120^{\circ} \mathrm{C}$ 吹扫样品表面 物理吸附的 $\mathrm{NH}_{3}$ 和弱吸附的 $\mathrm{NH}_{3}$ 后, 以 $20^{\circ} \mathrm{C} \cdot \mathrm{min}^{-1}$ 的升温速率升温至 $850^{\circ} \mathrm{C}$, 使吸附在样品表面的 $\mathrm{NH}_{3}$ 脱附. 催化剂表面元素的化学组成和化学态用 英国 VG Scientific 公司的 ESCALAB 210 型 X 射线 光电子能谱仪 (XPS) 分析. 使用 $\mathrm{Mg} K_{a}$ 射线(1253.6 $\mathrm{eV}, 300 \mathrm{~W})$, 在大约 $5 \times 10^{-9} \mathrm{~Pa}$ 下收集 XPS 样品. XPS 结合能用污染碳 C $1 s(284.6 \mathrm{eV})$ 进行校正, 以便于 催化剂与标准化合物的对比.

\subsection{B5-Ru 原子球棍模型绘制}

采用 FIZ/NIST Inorganic Crystal Structure Database Version 1.3.3 软件搜索空间构型为 P63/MMC 的 Ru 元素-ICSD \#43710, 将文件保存为 CIF 格式. 采用 DIAMOND Crystal and Molecular Structure Visualization 软件将此文件打开, 绘制 $3 \times 3 \times 3$ cells 的
$\mathrm{Ru}^{0}$ 晶体. 从中寻找满足以下两个条件的 5 个 $\mathrm{Ru}$ 原 子的集合体: (a)暴露出三叠层空位 (three-fold hollow site)和一个桥位(bridge site); (b)部分原子是低 坐标的表面原子 ${ }^{[2-3]}$.

\section{2 结果与讨论}

\section{1 催化剂的氨合成活性}

表 2 为氧化铝负载钉基催化剂的比表面积和氨 合成活性结果. 可以看出, 在不加入钡助剂时, $\mathrm{Ru} /$ $\mathrm{Al}_{2} \mathrm{O}_{3}-1$ 和 $\mathrm{Ru} / \mathrm{Al}_{2} \mathrm{O}_{3}-2$ 的氨合成活性基本相同, 在整 个反应温度范围内均较低. 随着 $\mathrm{BaO}$ 含量的增大, $\mathrm{Ru} / \mathrm{Al}_{2} \mathrm{O}_{3}-1$ 的氨合成活性先迅速增大, $\mathrm{Ba}-\mathrm{Ru}(3: 1) /$ $\mathrm{Al}_{2} \mathrm{O}_{3}-1$ 在 $450{ }^{\circ} \mathrm{C}$ 活性达到 $210.3 \mathrm{~mL} \cdot \mathrm{g}^{-1} \cdot \mathrm{h}^{-1}$, 继续增 大助剂钡含量至 $n(\mathrm{Ba}): n(\mathrm{Ru})=5: 1$ 时, 活性急剧下降 至 $\mathrm{Ru} / \mathrm{Al}_{2} \mathrm{O}_{3}-1$ 的水平. $\mathrm{BaO}$ 助剂对 $\mathrm{Ru} / \mathrm{Al}_{2} \mathrm{O}_{3}-2$ 的催 化活性的影响与此类似, 不同的是, 对于 $\mathrm{Al}_{2} \mathrm{O}_{3}-2$ 载 体, 当 $n(\mathrm{Ba}): n(\mathrm{Ru})=1: 1$ 时, 在 $450{ }^{\circ} \mathrm{C}$ 时活性达到最 大值 $221.2 \mathrm{~mL} \cdot \mathrm{g}^{-1} \cdot \mathrm{h}^{-1}$, 稍高于 $\mathrm{Ba}-\mathrm{Ru}(3: 1) / \mathrm{Al}_{2} \mathrm{O}_{3}-1$ 的 活性. 继续增大助剂钡含量至 $n(\mathrm{Ba}): n(\mathrm{Ru})=5: 1$ 时, 观察到活性下降至 $\mathrm{Ru} / \mathrm{Al}_{2} \mathrm{O}_{3}-2$ 的水平. 这表明, 存在 一个最佳的 $\mathrm{BaO}$ 含量值, 对表面性质不同的 $\gamma-\mathrm{Al}_{2} \mathrm{O}_{3}$ 载体, 这个最佳值也不同. 张新波等 ${ }^{[4]}$ 利用 $\mathrm{BaO}$ 改性 $\gamma-\mathrm{Al}_{2} \mathrm{O}_{3}$ 载体, 也发现氨合成活性随 $\mathrm{BaO}$ 含量的增大 而增大, 达到一个极大值后, 继续再加入 $\mathrm{BaO}$, 则造 成活性的急剧下降. 文献[15-16]的结果表明, $\mathrm{BaO}$ 助剂的作用主要分为中和酸性部位和传输电子给 $\mathrm{Ru}$ 两种, 当摩尔比 $n(\mathrm{Ba}): n(\mathrm{Ru})$ 为 2-4 时, 活性随钡 助剂含量的增大而呈上升趋势, 此时适量的 $\mathrm{BaO}$ 助 剂可以中和表面酸性和传递电子给 $\mathrm{Ru}^{0}$, 进一步增 加 $\mathrm{BaO}$ 含量造成催化剂表面的 $\mathrm{Ru}$ 活性中心被 $\mathrm{BaO}$ 层覆盖, 影响 $\mathrm{Ru}^{0}$-载体界面在反应气氛中的充分暴

表 $2 \mathrm{Al}_{2} \mathrm{O}_{3}$ 负载钉基催化剂的氨合成活性评价结果

Table 2 Rate of ammonia synthesis over the $\mathrm{Al}_{2} \mathrm{O}_{3}$ supported Ru catalysts

\begin{tabular}{lcrrr}
\hline \multirow{2}{*}{ Catalyst } & $n(\mathrm{Ba}): n(\mathrm{Ru})$ & \multicolumn{3}{c}{$x /\left(\mathrm{mL} \cdot \mathrm{g}^{-1} \cdot \mathrm{h}^{-1}\right)$} \\
\cline { 3 - 5 } & 0 & $425{ }^{\circ} \mathrm{C}$ & $450{ }^{\circ} \mathrm{C}$ & $475{ }^{\circ} \mathrm{C}$ \\
\hline $\mathrm{Ru} / \mathrm{Al}_{2} \mathrm{O}_{3}-1$ & $1: 1$ & 110.6 & 175.6 & 170.4 \\
$\mathrm{Ba}-\mathrm{Ru}(1: 1) / \mathrm{Al}_{2} \mathrm{O}_{3}-1$ & $3: 1$ & 180.6 & 210.3 & 186.9 \\
$\mathrm{Ba}-\mathrm{Ru}(3: 1) / \mathrm{Al}_{2} \mathrm{O}_{3}-1$ & $5: 1$ & 47.5 & 69.3 & 97.4 \\
$\mathrm{Ba}-\mathrm{Ru}(5: 1) / \mathrm{Al}_{2} \mathrm{O}_{3}-1$ & 0 & 46.3 & 72.4 & 85.1 \\
$\mathrm{Ru} / \mathrm{Al}_{2} \mathrm{O}_{3}-2$ & $1: 1$ & 139.0 & 221.2 & 214.4 \\
$\mathrm{Ba}-\mathrm{Ru}(1: 1) / \mathrm{Al}_{2} \mathrm{O}_{3}-2$ & $3: 1$ & 129.5 & 138.7 & 119.7 \\
$\mathrm{Ba}-\mathrm{Ru}(3: 1) / \mathrm{Al}_{2} \mathrm{O}_{3}-2$ & $5: 1$ & 38.0 & 60.2 & 96.9 \\
$\mathrm{Ba}-\mathrm{Ru}(5: 1) / \mathrm{Al}_{2} \mathrm{O}_{3}-2$ & & \multicolumn{3}{c}{} \\
\hline
\end{tabular}


露, 导致活性的下降.

\subsection{XRD 结果}

图 1 为氧化铝负载钉基催化剂经 $\mathrm{H}_{2}$ 于 $450{ }^{\circ} \mathrm{C}$ 还原后的 XRD 谱. 可以看出, 经 $\mathrm{H}_{2}$ 还原处理后所有 催化剂中均不存在 $\mathrm{Ru}^{0}$ 或 $\mathrm{RuO}_{2}$ 相, 这可能是由于金 属钉分散均匀或者以非晶形式存在 ${ }^{[17]}$. Aika 等 ${ }^{[10]}$ 认 为, 碱性助剂的加入可以增大钉活性物种的离子 势, 促进电子从 $\mathrm{Ru}^{0}$ 到 $\mathrm{N}_{2}$ 反键轨道的转移, 从而降低 $\mathrm{N}_{2}$ 解离吸附的活化能, 而这一步正是氨合成反应的 速率控制步骤. 加入 $\mathrm{BaO}$ 助剂后, $n(\mathrm{Ba}): n(\mathrm{Ru})=1: 1$ 时, 出现对应于 $\mathrm{BaCO}_{3}$ (JCPDS No. 05-0378) 相的新 衍射峰, 霍超等 ${ }^{\left[{ }^{[18}\right.}$ 认为这是由于经焙烧后生成的 $\mathrm{BaO}$ 晶粒较小, 碱性较强, 极易吸收空气中的 $\mathrm{CO}_{2}$, 从而生成 $\mathrm{BaCO}_{3}$ 晶相. 助剂钡含量增大至 $n(\mathrm{Ba})$ : $n(\mathrm{Ru})=3: 1$ 时, 除了 $\mathrm{BaCO}_{3}$ 的衍射峰, 同时还检测到 $\mathrm{BaAl}_{2} \mathrm{O}_{4}$ (JCPDS No. 17-0306) 衍射峰. $\mathrm{BaO}$ 在 $\mathrm{Al}_{2} \mathrm{O}_{3}$ 表面的单层分散阈值在 5\%-10\%(质量分数)之间, 当 $\mathrm{BaO}$ 含量超过 $10 \%, \mathrm{BaO}$ 与 $\mathrm{Al}_{2} \mathrm{O}_{3}$ 发生固相反应形 成对应于 $\mathrm{BaAl}_{2} \mathrm{O}_{4}$ 的新衍射峰 ${ }^{[4]}$.

图 1(b) 可见, 与 $\mathrm{Al}_{2} \mathrm{O}_{3}-1$ 相同, 沉淀法合成的
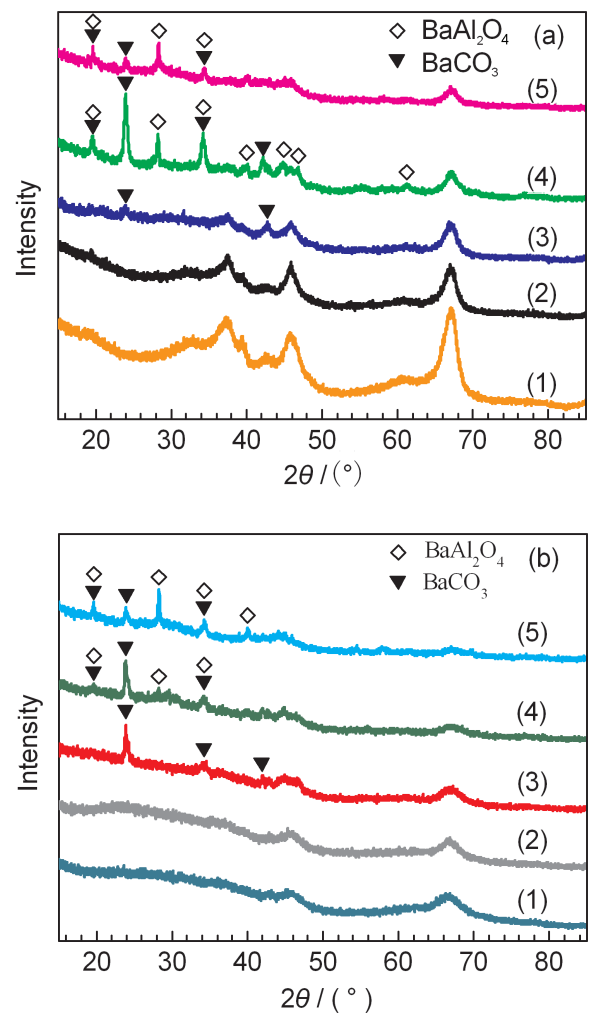

图 $1 \mathrm{Al}_{2} \mathrm{O}_{3}$ 负载钉基催化剂的 XRD 谱图

Fig.1 XRD patterns for the $\mathrm{Al}_{2} \mathrm{O}_{3}$ supported $\mathrm{Ru}$ catalysts (a) $\mathrm{Al}_{2} \mathrm{O}_{3}-1$, (b) $\mathrm{Al}_{2} \mathrm{O}_{3}-2$; (1) $\mathrm{Al}_{2} \mathrm{O}_{3}$, (2) $\mathrm{Ru} / \mathrm{Al}_{2} \mathrm{O}_{3}$, (3) $\mathrm{Ba}-\mathrm{Ru}(1: 1) / \mathrm{Al}_{2} \mathrm{O}_{3}$, (4) $\mathrm{Ba}-\mathrm{Ru}(3: 1) / \mathrm{Al}_{2} \mathrm{O}_{3}$, (5) $\mathrm{Ba}-\mathrm{Ru}(5: 1) / \mathrm{Al}_{2} \mathrm{O}_{3}$
$\mathrm{Al}_{2} \mathrm{O}_{3}-2$ 也为 $\gamma$ 型, 不过, 尽管晶型相同, 与 $\mathrm{Al}_{2} \mathrm{O}_{3}-1$ 载 体相比, $\mathrm{Al}_{2} \mathrm{O}_{3}-2$ 载体衍射峰较宽, 表明 $\mathrm{Al}_{2} \mathrm{O}_{3}-2$ 的晶 粒尺寸明显要小于 $\mathrm{Al}_{2} \mathrm{O}_{3}-1$ 的晶粒尺寸. 在 $n(\mathrm{Ba})$ : $n(\mathrm{Ru})=1: 1$ 时, $\mathrm{Ba}-\mathrm{Ru}(1: 1) / \mathrm{Al}_{2} \mathrm{O}_{3}-2$ 的 $\mathrm{BaCO}_{3}$ 相衍射峰 强度要强于 $\mathrm{Ba}-\mathrm{Ru}(1: 1) / \mathrm{Al}_{2} \mathrm{O}_{3}-1$ 的. 在 $n(\mathrm{Ba}): n(\mathrm{Ru})=$ 3:1 时, $\mathrm{Ba}-\mathrm{Ru}(3: 1) / \mathrm{Al}_{2} \mathrm{O}_{3}-1$ 的 $\mathrm{BaAl}_{2} \mathrm{O}_{4}$ 和 $\mathrm{BaCO}_{3}$ 相衍 射峰强度均强于 $\mathrm{Ba}-\mathrm{Ru}(3: 1) / \mathrm{Al}_{2} \mathrm{O}_{3}-2$ 的. 这可能是由 于 $\mathrm{BaO}$ 与不同来源的 $\mathrm{Al}_{2} \mathrm{O}_{3}$ 载体作用力的强弱不同 所致. 我们发现不同来源 $\gamma-\mathrm{Al}_{2} \mathrm{O}_{3}$ 负载钓基催化剂的 $\mathrm{XRD}$ 谱图均有共同点, 即 $\mathrm{BaO}$ 含量较低时, 在催化 剂中均不存在 $\mathrm{BaAl}_{2} \mathrm{O}_{4}$ 相, 而当 $\mathrm{BaO}$ 含量超过一定 值后, 出现了大量 $\mathrm{BaAl}_{2} \mathrm{O}_{4}$ 相, 对于不同来源的 $\gamma-\mathrm{Al}_{2} \mathrm{O}_{3}, \mathrm{BaAl}_{2} \mathrm{O}_{4}$ 相的强度不同, 这表明 $\mathrm{BaO}$ 与来源 不同的 $\gamma-\mathrm{Al}_{2} \mathrm{O}_{3}$ 的作用力存在差异.

\section{$2.3 \mathrm{~N}_{2}$ 低温物理吸附-脱附结果}

表 3 列出了 $\mathrm{Al}_{2} \mathrm{O}_{3}$ 载体及其负载钉基催化剂的 比表面积、孔容和平均孔径结果. $\mathrm{Al}_{2} \mathrm{O}_{3}-1$ 负载的钉 基催化剂的比表面积和孔体积均大于 $\mathrm{Al}_{2} \mathrm{O}_{3}-2$ 负载 的钉基催化剂的. 随着助剂钡含量的增加, $\mathrm{Ba}-\mathrm{Ru} /$ $\mathrm{Al}_{2} \mathrm{O}_{3}$ 催化剂比表面积和孔体积均呈现逐渐减小的 趋势. 图 2 为不同样品的 $\mathrm{N}_{2}$ 吸附-脱附等温线, $\mathrm{Al}_{2} \mathrm{O}_{3}$ 负载的钓催化剂具有典型的 IV 型等温线, 表明其具 有介孔结构, 在相对压力 $p / p_{0}$ 为 0.4-1.0范围内出现 因 $\mathrm{N}_{2}$ 的毛细管凝聚而产生的 $\mathrm{H} 2$ 型滞后环, 表明孔 径分布相对较窄, 孔形主要是蠕虫孔道 ${ }^{[19-20]}$. 图 3 为 $\mathrm{BJH}$ 法从 $\mathrm{N}_{2}$ 吸附-脱附等温线计算得到的 $\mathrm{Al}_{2} \mathrm{O}_{3}$ 负载 钉基催化剂孔径分布. 由脱附支可得 $\mathrm{Ru} / \mathrm{Al}_{2} \mathrm{O}_{3}-1$ 、 $\mathrm{Ba}-\mathrm{Ru}(1: 1) / \mathrm{Al}_{2} \mathrm{O}_{3}-1 、 \mathrm{Ba}-\mathrm{Ru}(3: 1) / \mathrm{Al}_{2} \mathrm{O}_{3}-1$ 和 $\mathrm{Ba}-\mathrm{Ru}(5$ : 1) $/ \mathrm{Al}_{2} \mathrm{O}_{3}-1$ 催化剂最可几孔径分布分别位于 3.2 和 $6.6 \mathrm{~nm} 、 3.2$ 和 $6.8 \mathrm{~nm} 、 3.5$ 和 $6.7 \mathrm{~nm} 、 3.4$ 和 $6.6 \mathrm{~nm}$, 而

表 3 载体和催化剂的织构性质

Table 3 Pore texture properties of $\mathrm{Al}_{2} \mathrm{O}_{3}$ supports and catalysts

\begin{tabular}{lcccc}
\hline Sample & $n(\mathrm{Ba}): n(\mathrm{Ru})$ & $S_{\mathrm{BET}} /\left(\mathrm{m}^{2} \cdot \mathrm{g}^{-1}\right)$ & $V_{\mathrm{p}} /\left(\mathrm{cm}^{3} \cdot \mathrm{g}^{-1}\right)$ & $D / \mathrm{nm}$ \\
\hline $\mathrm{Al}_{2} \mathrm{O}_{3}-1$ & 0 & 220 & 0.47 & 8.5 \\
$\mathrm{Ru} / \mathrm{Al}_{2} \mathrm{O}_{3}-1$ & 0 & 180 & 0.43 & 9.5 \\
$\mathrm{Ba}-\mathrm{Ru}(1: 1) / \mathrm{Al}_{2} \mathrm{O}_{3}-1$ & $1: 1$ & 161 & 0.35 & 8.6 \\
$\mathrm{Ba}-\mathrm{Ru}(3: 1) / \mathrm{Al}_{2} \mathrm{O}_{3}-1$ & $3: 1$ & 123 & 0.31 & 10.0 \\
$\mathrm{Ba}-\mathrm{Ru}(5: 1) / \mathrm{Al}_{2} \mathrm{O}_{3}-1$ & $5: 1$ & 86 & 0.24 & 11.0 \\
$\mathrm{Al}_{2} \mathrm{O}_{3}-2$ & 0 & 197 & 0.32 & 6.6 \\
$\mathrm{Ru} / \mathrm{Al}_{2} \mathrm{O}_{3}-2$ & 0 & 153 & 0.37 & 9.8 \\
$\mathrm{Ba}-\mathrm{Ru}(1: 1) / \mathrm{Al}_{2} \mathrm{O}_{3}-2$ & $1: 1$ & 118 & 0.21 & 7.2 \\
$\mathrm{Ba}-\mathrm{Ru}(3: 1) / \mathrm{Al}_{2} \mathrm{O}_{3}-2$ & $3: 1$ & 98 & 0.18 & 7.2 \\
$\mathrm{Ba}-\mathrm{Ru}(5: 1) / \mathrm{Al}_{2} \mathrm{O}_{3}-2$ & $5: 1$ & 58 & 0.10 & 6.5 \\
\hline
\end{tabular}

$S_{\mathrm{BET}}$ : surface area; $V_{\mathrm{p}}$ : pore volume; $D$ : pore diameter 

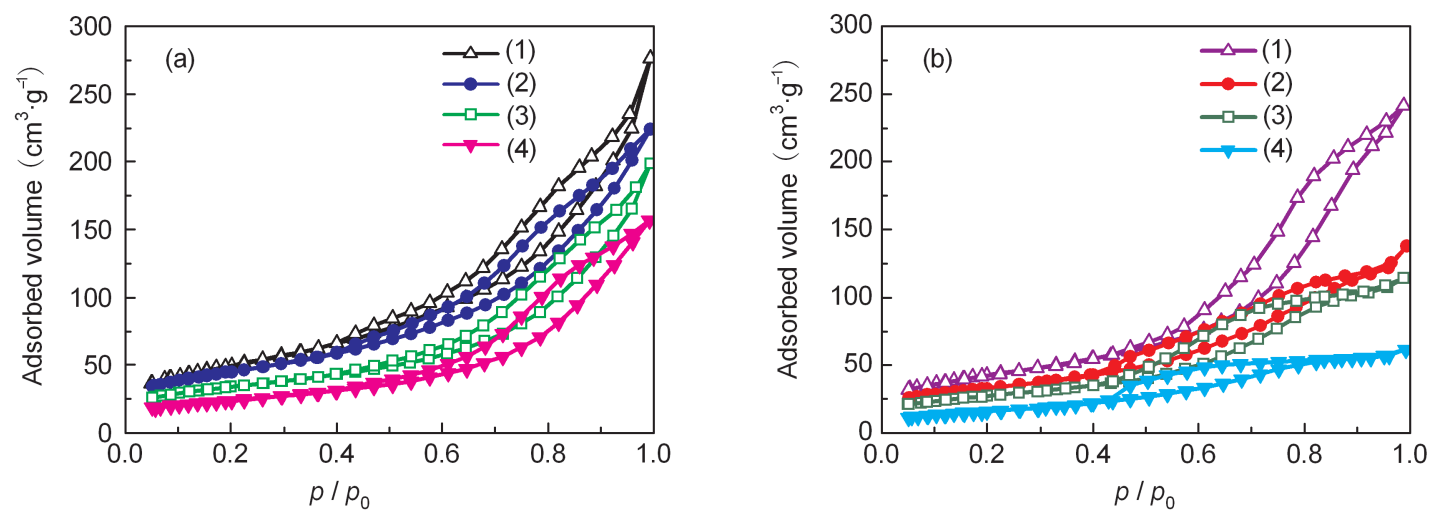

图 $2 \mathrm{Al}_{2} \mathrm{O}_{3}$ 负载钉基催化剂的 $\mathrm{N}_{2}$ 吸附-脱附等温线

Fig.2 $\mathrm{N}_{2}$ adsorption-desorption isotherms for the $\mathrm{Al}_{2} \mathrm{O}_{3}$ supported $\mathrm{Ru}$ catalysts

(a) $\mathrm{Al}_{2} \mathrm{O}_{3}-1$, (b) $\mathrm{Al}_{2} \mathrm{O}_{3}-2$; (1) $\mathrm{Ru} / \mathrm{Al}_{2} \mathrm{O}_{3}$, (2) $\mathrm{Ba}-\mathrm{Ru}(1: 1) / \mathrm{Al}_{2} \mathrm{O}_{3}$, (3) $\mathrm{Ba}-\mathrm{Ru}(3: 1) / \mathrm{Al}_{2} \mathrm{O}_{3}$, (4) $\mathrm{Ba}-\mathrm{Ru}(5: 1) / \mathrm{Al}_{2} \mathrm{O}_{3}$

根据吸附支计算, 孔径分布分别为 $3.5 、 3.8 、 5.5 、 6.1$ $\mathrm{nm}$, 吸附支与脱附支计算的 PSD 结果差异表明材料 中存在表面缺陷或特殊孔道结构, 吸脱附过程中造 成张力强度效应 (tensile strength effect) ${ }^{[20-22]}$. 与 $\mathrm{Al}_{2} \mathrm{O}_{3}-1$ 负载钓基催化剂类似, $\mathrm{Ru} / \mathrm{Al}_{2} \mathrm{O}_{3}-2 、 \mathrm{Ba}-\mathrm{Ru}(1$ : 1) $/ \mathrm{Al}_{2} \mathrm{O}_{3}-2 、 \mathrm{Ba}-\mathrm{Ru}(3: 1) / \mathrm{Al}_{2} \mathrm{O}_{3}-2$ 和 $\mathrm{Ba}-\mathrm{Ru}(5: 1) / \mathrm{Al}_{2} \mathrm{O}_{3}-2$ 催化剂由脱附支得到最可几孔径分布分别位于 3.4、 $5.4 、 7.4 \mathrm{~nm}, 3.5 \mathrm{~nm}, 3.4 、 4.0 \mathrm{~nm}, 3.3 \mathrm{~nm}$, 吸附支计算

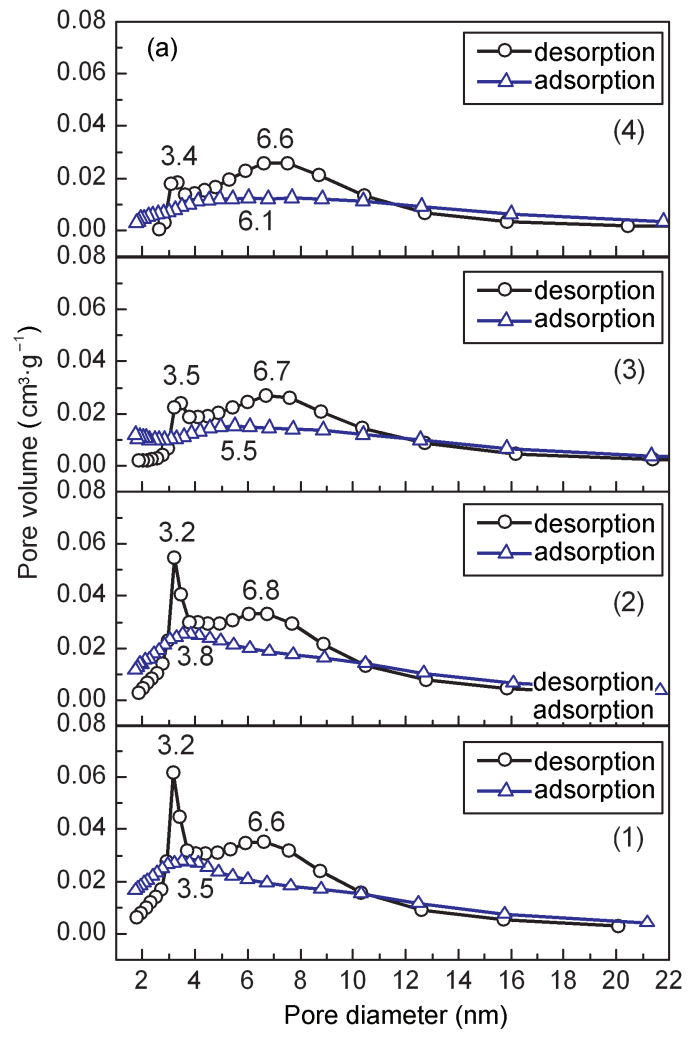

前 3 种催化剂的孔径分布分别为 7.6、4.6、5.5 nm, $\mathrm{Ba}-\mathrm{Ru}(5: 1) / \mathrm{Al}_{2} \mathrm{O}_{3}-2$ 催化剂孔道几乎全部堵塞, 因此 在吸附支上几乎看不到最可几孔径的分布, $\mathrm{BaO}$ 助 剂的过量加入对孔道的堵塞现象在一定程度上阻 滞了 $\mathrm{Ru}^{0}$-载体界面在反应气中的充分暴露.

\subsection{TEM 结果}

图 4 为于 $450{ }^{\circ} \mathrm{C}$ 用 $\mathrm{H}_{2}$ 还原后的 $\mathrm{Ba}-\mathrm{Ru}(1: 1) /$ $\mathrm{Al}_{2} \mathrm{O}_{3}-1$ 和 $\mathrm{Ba}-\mathrm{Ru}(1: 1) / \mathrm{Al}_{2} \mathrm{O}_{3}-2$ 催化剂的 TEM 照片.

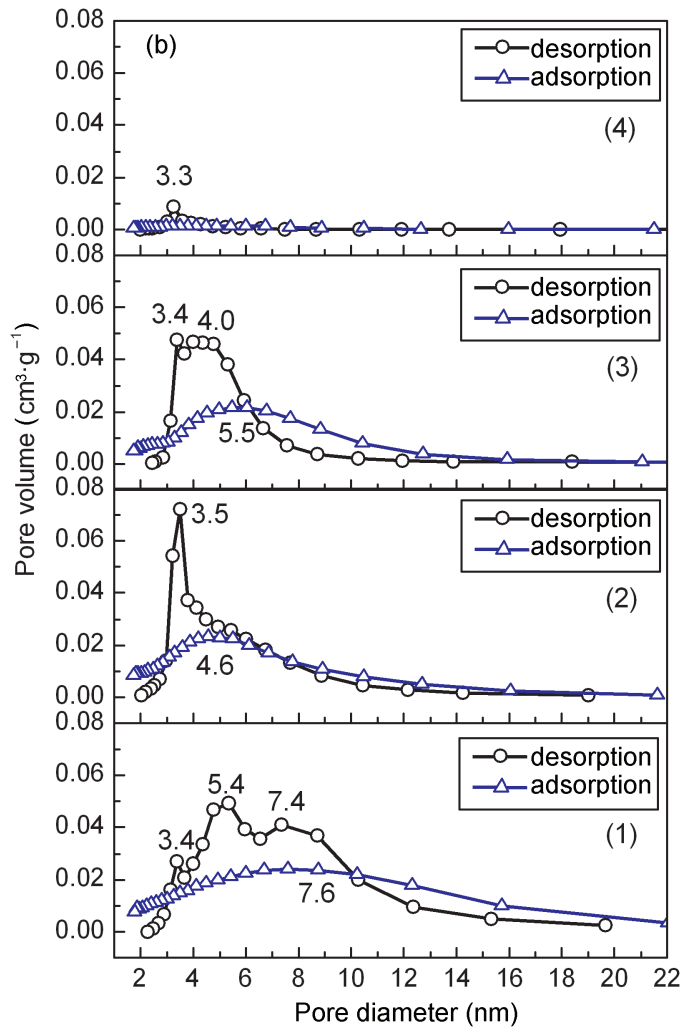

图 $3 \mathrm{Al}_{2} \mathrm{O}_{3}$ 负载钉基催化剂的孔径分布图

Fig.3 Pore size distribution of the $\mathrm{Al}_{2} \mathrm{O}_{3}$ supported $\mathrm{Ru}$ catalysts

(a) $\mathrm{Al}_{2} \mathrm{O}_{3}-1$, (b) $\mathrm{Al}_{2} \mathrm{O}_{3}-2$; (1) $\mathrm{Ru} / \mathrm{Al}_{2} \mathrm{O}_{3}$, (2) $\mathrm{Ba}-\mathrm{Ru}(1: 1) / \mathrm{Al}_{2} \mathrm{O}_{3}$, (3) $\mathrm{Ba}-\mathrm{Ru}(3: 1) / \mathrm{Al}_{2} \mathrm{O}_{3}$, (4) $\mathrm{Ba}-\mathrm{Ru}(5: 1) / \mathrm{Al}_{2} \mathrm{O}_{3}$ 


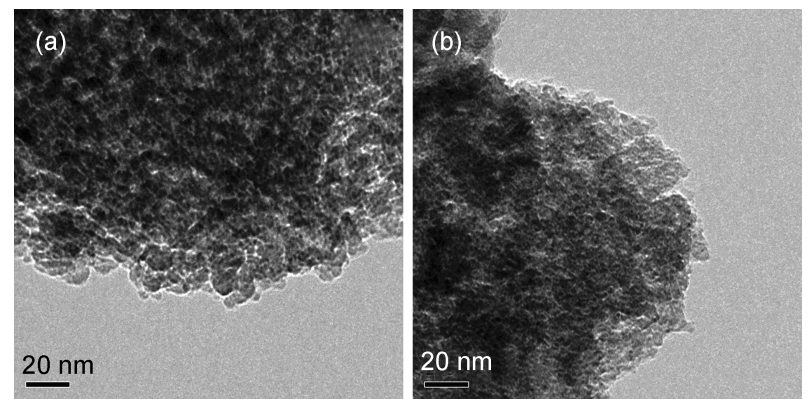

图 $4 \mathrm{Ba}-\mathrm{Ru}(1: 1) / \mathrm{Al}_{2} \mathrm{O}_{3}$ 催化剂的 TEM 照片

Fig.4 TEM images of $\mathrm{Ba}-\mathrm{Ru}(1: 1) / \mathrm{Al}_{2} \mathrm{O}_{3}$ catalysts

(a) $\mathrm{Ba}-\mathrm{Ru}(1: 1) / \mathrm{Al}_{2} \mathrm{O}_{3}-1$; (b) $\mathrm{Ba}-\mathrm{Ru}(1: 1) / \mathrm{Al}_{2} \mathrm{O}_{3}-2$

$\mathrm{Al}_{2} \mathrm{O}_{3}-1$ 和 $\mathrm{Al}_{2} \mathrm{O}_{3}-2$ 均存在大量蠕虫状孔道, 与 $\mathrm{N}_{2}$ 低 温物理吸附的结果一致(图 2). Ru 颗粒比较均匀地 分散在 $\mathrm{Al}_{2} \mathrm{O}_{3}$ 载体上, $\mathrm{Ru}_{3}(\mathrm{CO})_{12} / \mathrm{Al}_{2} \mathrm{O}_{3}$ 分散度比较高 的原因是由于 $\mathrm{Ru}_{3}(\mathrm{CO})_{12}$ 和 $\mathrm{Al}_{2} \mathrm{O}_{3}$ 表面的羟基反应, 生成了单原子层针式络合物 ${ }^{[16]}$. 随机选取 100 个 $\mathrm{Ru}$ 颗粒进行粒径分布统计, 结果表明, $\mathrm{Ru}$ 粒径均为 1-2 nm. 钉基催化剂表面上进行的氨合成反应是典 型的结构敏感反应, 密度泛函理论(DFT)计算表明, 对 $\mathrm{N}_{2}$ 的解离和氨合成反应最具有活性的位置是满 足两个条件的 5 个 $\mathrm{Ru}$ 原子的集合体(如 1.5 部分所 述 $)^{[2]}$. B5 位的数量与 $\mathrm{Ru}$ 粒子大小有密切关系(图 5), Jacobsen 等 ${ }^{[23]}$ 认为 $\mathrm{Ru}$ 粒径在 1.5-2.0 nm 时, 较易形 成 B5 结构反应活性中心, 有利于活性的提高. 随着 $\mathrm{Ru}$ 晶粒的长大, 低坐标表面原子所占比率会减少, B5 中心数量下降. $\mathrm{Al}_{2} \mathrm{O}_{3}$ 负载钉基催化剂在形成 B5 活性中心方面是有利的, 而其活性的差异则主要是 由其体系的电子性质影响所致.

\section{$2.5 \mathrm{H}_{2}$-TPR 结果}

图 6(1-4)为 $\mathrm{Al}_{2} \mathrm{O}_{3}-1$ 负载钉基催化剂的 $\mathrm{TPR}$ 谱. $\mathrm{Ru} / \mathrm{Al}_{2} \mathrm{O}_{3}-1$ 催化剂呈现典型的 “双峰”还原过程. 研

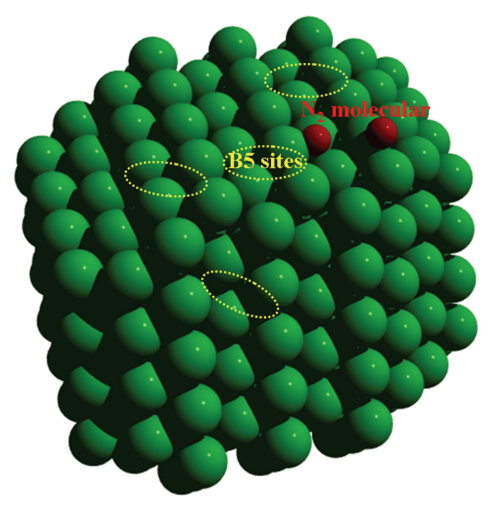

图 $5 \mathrm{Ru}$ 晶体中 $\mathrm{N}_{2}$ 吸附解离的 $\mathrm{B} 5$ 位示意图

Fig.5 Ball model illustrating B5 sites on the side facets of Ru crystallites
究表明, 钉催化剂上存在两种 $\mathrm{Ru}$ 物种的还原峰, 低 温峰为呈现高分散状态的 $R u$ 粒子或表相的 $R u$ 物 种 $-\mathrm{RuO}_{x}$ 的还原峰, 而高温峰为体相 $\mathrm{Ru}$ 粒子 $-\mathrm{RuO}_{2}$ 的还原峰, 前者的峰温较低 $\left(150-180{ }^{\circ} \mathrm{C}\right)$, 而后者的 还原温度较高 $\left(300{ }^{\circ} \mathrm{C}\right)^{[24]}$. $\mathrm{Ru} / \mathrm{Al}_{2} \mathrm{O}_{3}-1$ 催化剂的 $\mathrm{Ru}$ 物 种在 92 和 $325^{\circ} \mathrm{C}$ 分别发生还原; $\mathrm{Ba}-\mathrm{Ru}(1: 1) / \mathrm{Al}_{2} \mathrm{O}_{3}-1$ 催化剂的 $\mathrm{Ru}$ 物种在 $25-125^{\circ} \mathrm{C}$ 的范围内几乎已经 被全部还原, 在 $175-475^{\circ} \mathrm{C}$ 的温度范围出现宽的高 温还原峰, 强度较小. 这表明适量 $\mathrm{BaO}$ 助剂的加入 促进了钓催化剂的还原表现, $n(\mathrm{Ba}): n(\mathrm{Ru})=1: 1$ 时, 助 剂 $\mathrm{BaO}$ 在载体表面呈单分散状态, $\mathrm{Ru}-\mathrm{BaO}$ 界面在 反应气氛中便于暴露, $\mathrm{Ru}$ 物种还原容易. $\mathrm{Ba}-\mathrm{Ru}(3$ : 1) $/ \mathrm{Al}_{2} \mathrm{O}_{3}-1$ 催化剂在整个还原温度范围内出现三个 还原峰, 峰温在 140 和 $312^{\circ} \mathrm{C}$ 的还原峰分别为 $\mathrm{Ru}$ 物 种的低温、高温还原峰, 与不加助剂的 $\mathrm{Ru} / \mathrm{Al}_{2} \mathrm{O}_{3}-1$ 相 比, 过量助剂 $\mathrm{BaO}$ 的加入使 $\mathrm{Ru}$ 物种的还原变得更 加困难 ${ }^{[25]}$, 这是由于当 $n(\mathrm{Ba}): n(\mathrm{Ru})$ 为 $3: 1$ 和 5:1 时, 助 剂量超过了单层分布的量, 造成大量 $\mathrm{BaO}$ 助剂附着 在 $\mathrm{Ru}$ 和载体表面, $\mathrm{Ru}-\mathrm{BaO}$ 界面不能在反应气氛中

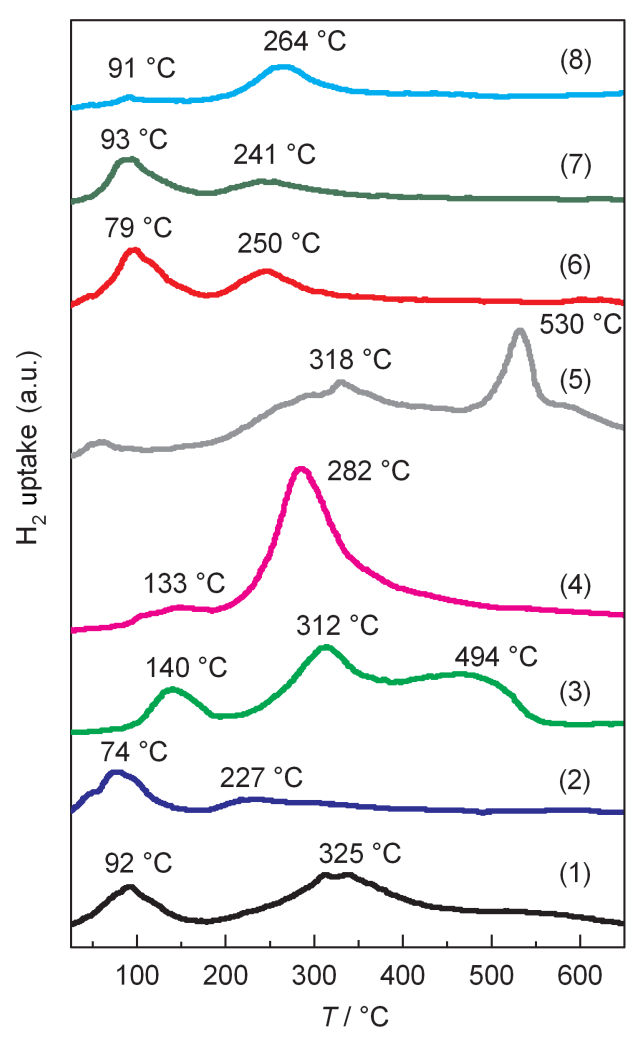

图 $6 \mathrm{Al}_{2} \mathrm{O}_{3}$ 负载钉基催化剂的 $\mathrm{H}_{2}$-TPR 谱

Fig.6 $\mathrm{H}_{2}$-TPR patterns of the $\mathrm{Al}_{2} \mathrm{O}_{3}$ supported $\mathrm{Ru}$ catalysts

(1) $\mathrm{Ru} / \mathrm{Al}_{2} \mathrm{O}_{3}-1$; (2) $\mathrm{Ba}-\mathrm{Ru}(1: 1) / \mathrm{Al}_{2} \mathrm{O}_{3}-1$; (3) $\mathrm{Ba}-\mathrm{Ru}(3: 1) / \mathrm{Al}_{2} \mathrm{O}_{3}-1$;

(4) $\mathrm{Ba}-\mathrm{Ru}(5: 1) / \mathrm{Al}_{2} \mathrm{O}_{3}-1$; (5) Ru/ $/ \mathrm{Al}_{2} \mathrm{O}_{3}-2$; (6) $\mathrm{Ba}-\mathrm{Ru}(1: 1) / \mathrm{Al}_{2} \mathrm{O}_{3}-2$;

(7) $\mathrm{Ba}-\mathrm{Ru}(3: 1) / \mathrm{Al}_{2} \mathrm{O}_{3}-2 ;(8) \mathrm{Ba}-\mathrm{Ru}(5: 1) / \mathrm{Al}_{2} \mathrm{O}_{3}-2$ 
暴露, 造成其还原困难, 还原峰温度上升, 过量的 $\mathrm{BaO}$ 助剂阻滞了 $\mathrm{Ru}$ 物种的还原过程 ${ }^{[2]}$. $\mathrm{Ba}-\mathrm{Ru}(3: 1) /$ $\mathrm{Al}_{2} \mathrm{O}_{3}-1$ 催化剂的第三个宽的肩峰出现在 $400-550{ }^{\circ} \mathrm{C}$ 温度区间, XRD 结果 (图 1a(4)) 表明 $\mathrm{Ba}-\mathrm{Ru}(3: 1) /$ $\mathrm{Al}_{2} \mathrm{O}_{3}-1$ 催化剂中存在大量 $\mathrm{BaCO}_{3}$ 物相, 因此, 这个 还原峰是由于 $\mathrm{BaO}$ 助剂与空气作用生成的 $\mathrm{BaCO}_{3}$ 的热分解造成. $\mathrm{BaCO}_{3}$ 是碱土金属碳酸盐中最稳定 的碳酸盐, 在空气中的理论分解温度达到 $1287^{\circ} \mathrm{C}$. 霍超等 ${ }^{[26]}$ 系统研究了氢气还原气氛下催化剂中 $\mathrm{BaCO}_{3}$ 的热稳定性, 他们认为, 在 $\mathrm{Ru}^{0}$ 表面存在氢溢 流现象, $\mathrm{H}_{2}$ 在 $\mathrm{Ru}$ 作用下解离为氢原子, 从 $\mathrm{Ru}^{0}$ 表面 溢流至载体表面, 从而有效地促进了 $\mathrm{BaCO}_{3}$ 的低温 分解过程. $\mathrm{BaO}$ 是钓基氨合成催化剂的有效助剂, Hansen 等 ${ }^{[27]}$ 认为靠近于 $\mathrm{Ru}$ 晶体 $\mathrm{B} 5$ 活性位的 $\mathrm{BaO}$ 是 催化剂的电子助剂, 可以极大地促进氨合成活性. 氨合成活性结果也表明, $\mathrm{Ba}-\mathrm{Ru}(3: 1) / \mathrm{Al}_{2} \mathrm{O}_{3}-1$ 催化剂 在 $450{ }^{\circ} \mathrm{C}, 5 \mathrm{MPa}, 5000 \mathrm{~h}^{-1}$ 反应条件下活性达到 $210.3 \mathrm{~mL} \cdot \mathrm{g}^{-1} \cdot \mathrm{h}^{-1}$, 明显高于其他 $\mathrm{Al}_{2} \mathrm{O}_{3}-1$ 负载钓基催 化剂的氨合成活性. Ba- $\mathrm{Ru}(5: 1) / \mathrm{Al}_{2} \mathrm{O}_{3}-1$ 样品在 $282^{\circ} \mathrm{C}$ 出现较强的体相 $\mathrm{RuO}_{2}$ 还原峰, 再次证实 $\mathrm{BaO}$ 助剂的 过量加入确实对 $\mathrm{Ru}$ 物种的还原过程有阻滞作用.

图 6(5-8)为 $\mathrm{Al}_{2} \mathrm{O}_{3}$-2 负载钉基催化剂的 $\mathrm{TPR}$ 谱. 与 $\mathrm{Ru} / \mathrm{Al}_{2} \mathrm{O}_{3}-1$ 相同, $\mathrm{Ru} / \mathrm{Al}_{2} \mathrm{O}_{3}-2$ 样品的还原过程也分 为两步还原, 然而, 很明显其还原峰温度 (318 和 $530{ }^{\circ} \mathrm{C}$ )要高于前者, 这可能是由于 $\mathrm{Al}_{2} \mathrm{O}_{3}-2$ 与 $\mathrm{Ru}$ 物 种的作用程度较 $\mathrm{Al}_{2} \mathrm{O}_{3}-1$ 更强, 造成还原温度升高. 与 $\mathrm{Al}_{2} \mathrm{O}_{3}-1$ 负载钉基催化剂的还原表现不尽相同, $n(\mathrm{Ba}): n(\mathrm{Ru})$ 为 $1: 1$ 和 3:1 时, $\mathrm{BaO}$ 助剂的加入造成 $\mathrm{Ba}-\mathrm{Ru} / \mathrm{Al}_{2} \mathrm{O}_{3}-2$ 的还原峰向低温方向移动, $\mathrm{Ba}-\mathrm{Ru}(1$ : 1) $/ \mathrm{Al}_{2} \mathrm{O}_{3}-2$ 峰温分别为 79 和 $250{ }^{\circ} \mathrm{C}, \mathrm{Ba}-\mathrm{Ru}(3: 1) /$ $\mathrm{Al}_{2} \mathrm{O}_{3}-2$ 峰温分别为 93 和 $241^{\circ} \mathrm{C}$, 且大部分均在低温 时被还原. 这表明 $\mathrm{BaO}$ 在 $\mathrm{Al}_{2} \mathrm{O}_{3}$-2 上单分散分布的量 要大于在 $\mathrm{Al}_{2} \mathrm{O}_{3}-1$ 上的. $n(\mathrm{Ba}): n(\mathrm{Ru})=5: 1$ 时, $\mathrm{Ba}-\mathrm{Ru}(5$ : 1) $/ \mathrm{Al}_{2} \mathrm{O}_{3}-2$ 峰温分别为 91 和 $264{ }^{\circ} \mathrm{C}$, 且大部分在高 温时被还原, 这表明此时 $\mathrm{BaO}$ 在催化剂表面并非单 分散状态, 而是覆盖在 $\mathrm{Ru}$ 物种和载体表面, 造成 $\mathrm{Ru}-\mathrm{BaO}$ 界面难于接触反应气, 还原困难. 活性评价 结果表明 $\mathrm{Ba}-\mathrm{Ru}(1: 1) / \mathrm{Al}_{2} \mathrm{O}_{3}-2$ 催化剂的活性 $\left(450^{\circ} \mathrm{C}\right.$, $\left.221.2 \mathrm{~mL} \cdot \mathrm{g}^{-1} \cdot \mathrm{h}^{-1}\right)$ 稍高于 $\mathrm{Ba}-\mathrm{Ru}(3: 1) / \mathrm{Al}_{2} \mathrm{O}_{3}-2$ 的 $\left(450{ }^{\circ} \mathrm{C}, 138.7 \mathrm{~mL} \cdot \mathrm{g}^{-1} \cdot \mathrm{h}^{-1}\right)$ 的活性, 这表明钉基催化 剂的氨合成活性与 $\mathrm{Ru}$ 物种的还原性质密切相关, 不 仅是表相 $\mathrm{RuO}_{x}$ 物种的还原, 体相 $\mathrm{RuO}_{2}$ 的还原也对 反应有影响. $\mathrm{Ba}-\mathrm{Ru}(5: 1) / \mathrm{Al}_{2} \mathrm{O}_{3}-2$ 的钓物种主要以体
相 $\mathrm{RuO}_{2}$ 形式被还原, 氨合成活性较低. $\mathrm{Dahl}$ 等 ${ }^{[28]}$ 认 为 $\mathrm{B} 5$ 位是对 $\mathrm{N}_{2}$ 的解离和氨合成反应最具有活性的 $\mathrm{Ru}$ 原子排布方式, 而 $\mathrm{B} 5$ 位的丰度则取决于 $\mathrm{Ru}$ 粒子 的形状和尺寸大小, Ru粒径与还原温度相关, 因此, $\mathrm{Ru}$ 物种的还原性质对催化剂的氨合成活性有较大 影响.

\section{$2.6 \mathrm{NH}_{3}$-TPD 结果}

在氨合成反应中, $\mathrm{N} \equiv \mathrm{N}$ 键的断裂是非常困难 的, 碱金属或碱土金属等供电子助催化剂的加入能 够提供电子给活性组分 $\mathrm{Ru}$, 削弱 $\mathrm{N}_{2}$ 分子内的键能, 增强 $\mathrm{N}_{2}$ 的解离吸附, 从而提高氨合成反应速度. 氨 合成活性与载体的碱性成正比, 即碱性越大, 活性越 高 ${ }^{[2-3,25]} \cdot \mathrm{Al}_{2} \mathrm{O}_{3}$ 是一种常用的催化剂载体, 是强的 Lewis 酸, 室温下 $\mathrm{Al}_{2} \mathrm{O}_{3}$ 表面为 $\mathrm{OH}^{-}$所覆盖, 呈非酸 性, 当温度升高时, 表面的 $\mathrm{OH}^{-}$与相邻的 $\mathrm{H}$ 生成 $\mathrm{H}_{2} \mathrm{O}$ 分子而被除去, 形成不饱和配位的 $\mathrm{Al}^{3+}$, 成为 Lewis 酸中心, 一般在 $>300{ }^{\circ} \mathrm{C}$ 才能观察到酸性, 而 Lewis 酸吸附水气后形成具有弱 Brønsted 特征的质子酸部 位 ${ }^{[29]}$. 除了酸特征外, 脱水过程中形成的带负电荷的 $\mathrm{O}^{-}$形成了碱性部位. 由于酸性部位阻滞了氨合成反 应过程中载体或者助剂到 $\mathrm{Ru}$ 的电子传输, 因此, 添 加碱性助剂是必要的. 我们利用 $\mathrm{NH}_{3}-\mathrm{TPD}$ 考察了纯 氧化铝载体和添加碱性助剂 $\mathrm{BaO}$ 的 $\mathrm{Ba}-\mathrm{Ru} / \mathrm{Al}_{2} \mathrm{O}_{3}$ 催 化剂的表面酸性性质. 图 7 为 $\mathrm{Al}_{2} \mathrm{O}_{3}$ 载体的 $\mathrm{NH}_{3}-\mathrm{TPD}$ 谱图, $\mathrm{Al}_{2} \mathrm{O}_{3}-1$ 在整个 $\mathrm{NH}_{3}$ 脱附温度范围内明显出现 两个峰, 峰顶温度分别为 167 和 $568{ }^{\circ} \mathrm{C}$, 表明在氧化 铝表面明显存在弱酸性位和强酸性位. 脱附曲线在 从低温到高温过程中没有回到基线, 表明表面存在 一定数量的中等强度的酸性位 ${ }^{[30]}$. Somorjai 等 ${ }^{\left[{ }^{[1]}\right.}$ 认为 $\mathrm{Al}_{2} \mathrm{O}_{3}$ 表面存在 3 类不同配位数的 $\mathrm{Al}$ 原子: 高配位数 $\mathrm{Al}$ 原子(低表面能, 弱酸中心)、中等配位数 $\mathrm{Al}$ 原子

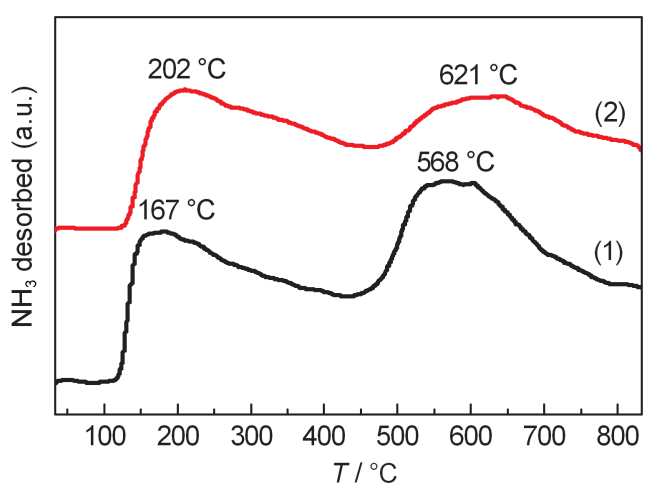

图 $7 \mathrm{Al}_{2} \mathrm{O}_{3}$ 的 $\mathrm{NH}_{3}-\mathrm{TPD}$ 谱 Fig.7 $\mathrm{NH}_{3}$-TPD spectra of $\mathrm{Al}_{2} \mathrm{O}_{3}$ (1) $\mathrm{Al}_{2} \mathrm{O}_{3}-1 ;(2) \mathrm{Al}_{2} \mathrm{O}_{3}-2$ 

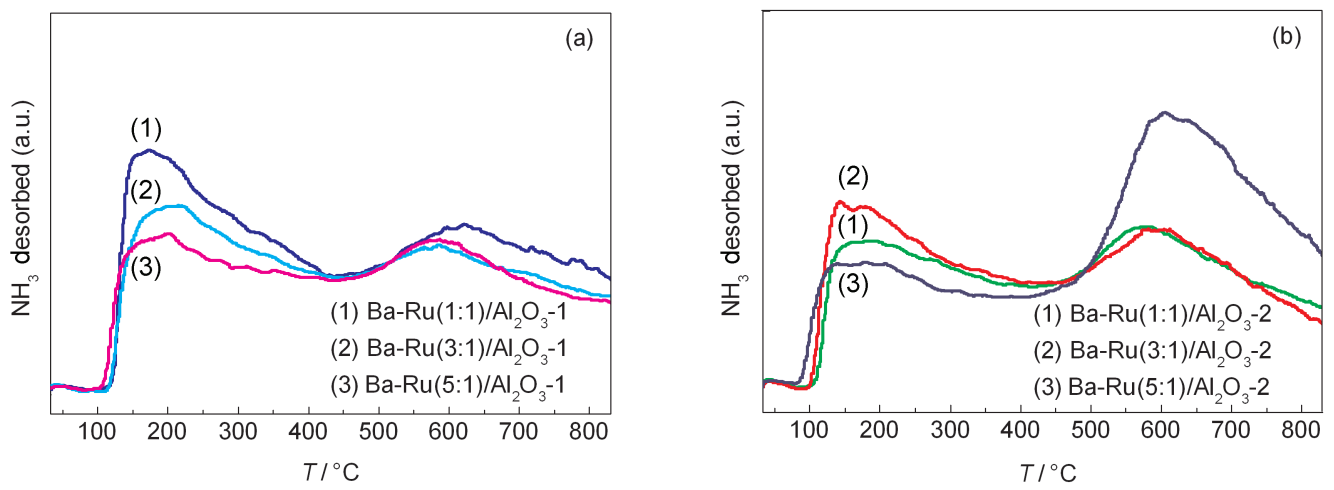

图 $8 \mathrm{Al}_{2} \mathrm{O}_{3}$ 负载钉基催化剂的 $\mathrm{NH}_{3}$-TPD 谱

Fig.8 $\mathrm{NH}_{3}$-TPD spectra of the $\mathrm{Al}_{2} \mathrm{O}_{3}$ supported $\mathrm{Ru}$ catalysts

(a) $\mathrm{Ba}-\mathrm{Ru} / \mathrm{Al}_{2} \mathrm{O}_{3}-1$; (b) $\mathrm{Ba}-\mathrm{Ru} / \mathrm{Al}_{2} \mathrm{O}_{3}-2$

(中等表面能, 中等酸中心)和低配位数 $\mathrm{Al}$ 原子(高表 面能, 强酸中心). $\mathrm{OH}^{-}$分别与酸性不同的酸中心结 合, 从而形成三类酸强度各不相同的表面一 $\mathrm{OH}$.

以 $\mathrm{NH}_{3}$ 为吸附质的 $\mathrm{Al}_{2} \mathrm{O}_{3}$ 的 TPD 谱图, 虽然因为 $\mathrm{Al}_{2} \mathrm{O}_{3}$ 的制备方法不同而呈现不尽相同的谱图, 但其 峰形弥散又相互重叠则是共同的特点, 表明酸强度 分布并不均匀. 与 $\mathrm{Al}_{2} \mathrm{O}_{3}-1$ 相比, $\mathrm{Al}_{2} \mathrm{O}_{3}-2$ 的 $\mathrm{NH}_{3}-\mathrm{TPD}$ 谱图轮廓相同, 不同的是其脱附峰峰顶温度(202 和 $621^{\circ} \mathrm{C}$ )较前者更高. 这表明沉淀法合成的 $\mathrm{Al}_{2} \mathrm{O}_{3}-2$ 的 表面酸强度较 $\mathrm{Al}_{2} \mathrm{O}_{3}-1$ 更强. $\mathrm{NH}_{3}-\mathrm{TPD}$ 峰面积的分析 数据表明, $\mathrm{Al}_{2} \mathrm{O}_{3}-2$ 的 Lewis 酸性位数量明显比 $\mathrm{Al}_{2} \mathrm{O}_{3}-1$ 的多. 我们考察了 $\mathrm{BaO}$ 助剂添加量对不同来 源 $\mathrm{Ba}-\mathrm{Ru} / \mathrm{Al}_{2} \mathrm{O}_{3}$ 体系酸性的影响(图 8), $\mathrm{BaO}$ 助剂在 $\mathrm{Ba}-\mathrm{Ru} / \mathrm{Al}_{2} \mathrm{O}_{3}-1$ 体系中存在状态随添加量而不同, $n(\mathrm{Ba}): n(\mathrm{Ru})=1: 1$ 时, 主要以 $\mathrm{BaCO}_{3}$ 形态存在; $n(\mathrm{Ba})$ : $n(\mathrm{Ru})$ 为 $3: 1$ 和 $5: 1$ 时, 主要以 $\mathrm{BaCO}_{3}$ 和 $\mathrm{BaAl}_{2} \mathrm{O}_{4}$ 形态 存在. 由图 8(a) 可以看出, 体系弱酸性位数量随助剂 的添加量增大而减小, 体系总酸性位数量也是逐渐
减少, 这表明 $\mathrm{BaO}$ 助剂的添加造成体系酸性下降. $\mathrm{BaO}$ 助剂添加量对 $\mathrm{Ba}-\mathrm{Ru} / \mathrm{Al}_{2} \mathrm{O}_{3}-2$ 体系酸性的影响 则不相同, 体系弱酸性位数量先增加后减少, 在 $n(\mathrm{Ba}): n(\mathrm{Ru})=3: 1$ 时达到极大值, 而强酸性位数量则 在 $n(\mathrm{Ba}): n(\mathrm{Ru})=5: 1$ 时达到极大值 (图 8(b)). $\mathrm{BaO}$ 助剂 添加的结果是体系总酸性位数量增大, $n(\mathrm{Ba}): n(\mathrm{Ru})=$ $5: 1$ 时总酸性达到极大值. $\mathrm{BaO}$ 的加入没有降低体系 酸性, 相反, 体系酸性随 $\mathrm{BaO}$ 的加入而增大, 这种现 象可能是由于 $\mathrm{Ru} / \mathrm{Al}_{2} \mathrm{O}_{3}$ 催化剂对 $\mathrm{NH}_{3}$ 的催化分解作 用所致, $\mathrm{Nagai}$ 等 ${ }^{[32}$ 认为, $\mathrm{Ru} / \mathrm{Al}_{2} \mathrm{O}_{3}$ 样品的 $\mathrm{NH}_{3}$ - $\mathrm{TPD}$ 高温峰归因为 $\mathrm{NH}_{3}$ 分解成 $\mathrm{N}_{2}$ 和 $\mathrm{H}_{2}$, 而对 $\mathrm{Ru}$ 的不同 处理过程会造成 $\mathrm{NH}_{3}$ 分解作用的强弱区别, 这可能 就是我们在 $\mathrm{Ba}-\mathrm{Ru} / \mathrm{Al}_{2} \mathrm{O}_{3}-1$ 体系中并没有观察这种 现象的原因. 图 9 为氨合成活性与体系总酸量随助 剂添加量的变化曲线, 对 $\mathrm{Ba}-\mathrm{Ru} / \mathrm{Al}_{2} \mathrm{O}_{3}-1$ 体系来说, 活性随助剂 $\mathrm{BaO}$ 添加量呈 “火山” 曲线, 而总酸性则 单调减小, 这可能是由于体系酸性、比表面积和氨 合成活性之间存在最优比例(图 9(a)). Ba- $\mathrm{Ru} / \mathrm{Al}_{2} \mathrm{O}_{3}-2$
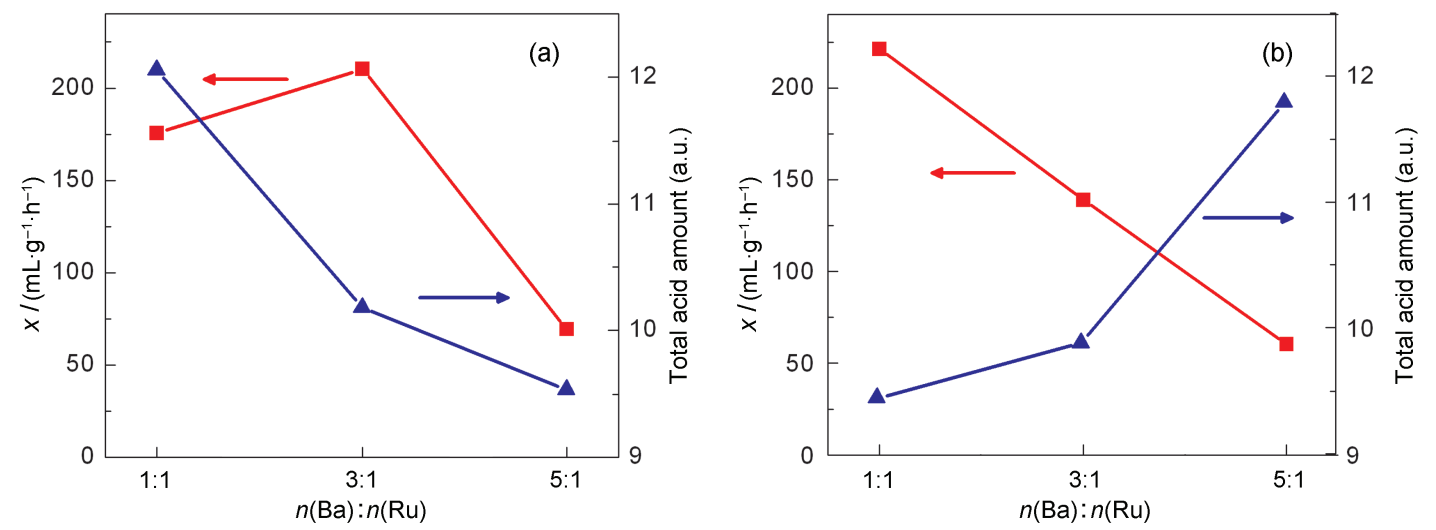

图 $9 \mathrm{Ba}-\mathrm{Ru} / \mathrm{Al}_{2} \mathrm{O}_{3}$ 催化剂氨合成活性与酸总量随 $\mathrm{BaO}$ 助剂添加量的变化曲线

Fig.9 Curves of ammonia formation rate and total acid amount of $\mathrm{Ba}-\mathrm{Ru}_{1} / \mathrm{Al}_{2} \mathrm{O}_{3}$ vs $\mathrm{Ba} / \mathrm{Ru}$ molar ratio (a) $\mathrm{Ba}-\mathrm{Ru} / \mathrm{Al}_{2} \mathrm{O}_{3}-1$; (b) $\mathrm{Ba}-\mathrm{Ru} / \mathrm{Al}_{2} \mathrm{O}_{3}-2$ 

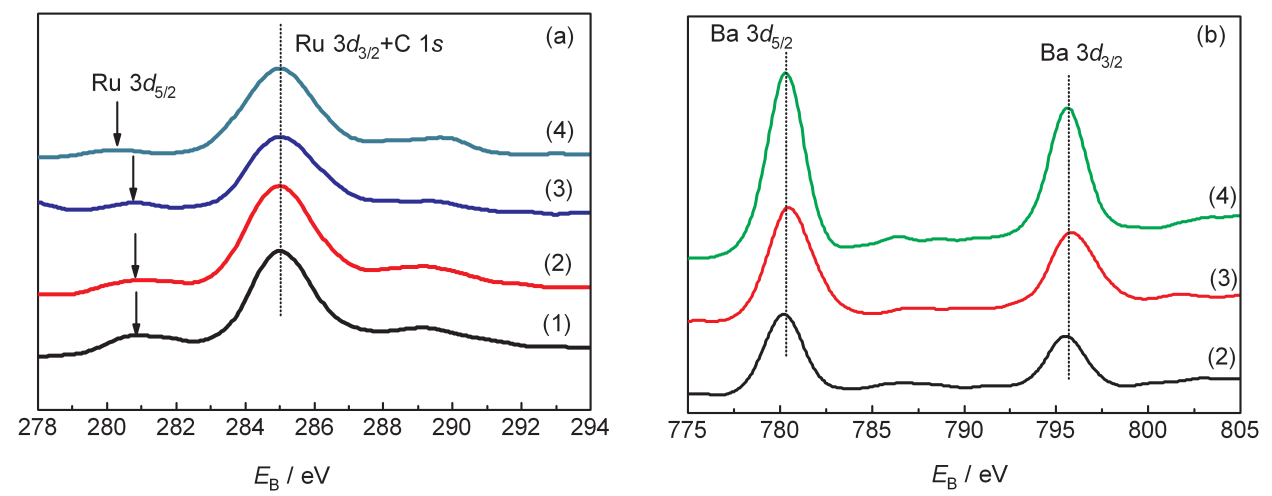

图 $10 \mathrm{Ba}-\mathrm{Ru} / \mathrm{Al}_{2} \mathrm{O}_{3}-2$ 催化剂的 $\mathrm{Ru} 3 d$ 和 $\mathrm{Ba} 3 d \mathrm{XPS}$ 谱

Fig.10 XPS patterns of $\mathrm{Ru} 3 d$ and $\mathrm{Ba} 3 d$ for $\mathrm{Ba}-\mathrm{Ru} / \mathrm{Al}_{2} \mathrm{O}_{3}-2$ catalysts

(a) Ru 3d, (b) Ba 3d; (1) Ru/ $\mathrm{Al}_{2} \mathrm{O}_{3}-2$, (2) Ba-Ru(1:1)/ $/ \mathrm{Al}_{2} \mathrm{O}_{3}-2$, (3) $\mathrm{Ba}-\mathrm{Ru}(3: 1) / \mathrm{Al}_{2} \mathrm{O}_{3}-2$, (4) $\mathrm{Ba}-\mathrm{Ru}(5: 1) / \mathrm{Al}_{2} \mathrm{O}_{3}-2$

体系氨合成活性与体系总酸性成反比(图9(b)), 这表 明对于 $\mathrm{Al}_{2} \mathrm{O}_{3}-2$ 负载钉基催化剂, 氨合成活性与体系 碱性是成正比的, 体系碱性对氨合成活性的影响要 强于比表面对其的影响.

\subsection{XPS 结果}

图 10 为 $\mathrm{Ba}-\mathrm{Ru} / \mathrm{Al}_{2} \mathrm{O}_{3}-2$ 催化剂的 XPS 谱图. 由 于轨道-耦合作用, $\mathrm{Ru}$ 的谱图包含 $3 d_{3 / 2}$ 和 $3 d_{5 / 2}$ 两个 峰, $\mathrm{Ru} 3 d_{3 / 2}$ 与 $\mathrm{C} 1 s$ 峰重叠, 碳污染来自催化剂表面 与空气中 $\mathrm{CO}_{2}$ 的化学吸附或者表面键合作用, 因 此, 一般以 $\mathrm{Ru} 3 d_{5 / 2}$ 峰为研究对象 ${ }^{[9]}$. 如图 10(a)所示, $\mathrm{Ru} / \mathrm{Al}_{2} \mathrm{O}_{3}$ 催化剂中 $\mathrm{Ru} 3 d_{5 / 2}$ 峰位于 $280.7 \mathrm{eV}$, 随着 $\mathrm{BaO}$ 助剂含量的增加, $\mathrm{Ru} 3 d_{5 / 2}$ 峰位依次为 280.7 、 280.7 和 $280.2 \mathrm{eV} . \mathrm{Ru}^{0} 3 d_{5 / 2}$ 为 $280.0 \mathrm{eV}, \mathrm{RuO}_{2} 3 d_{5 / 2}$ 为 $280.6 \mathrm{eV}$, 显然, $\mathrm{Ru} / \mathrm{Al}_{2} \mathrm{O}_{3} 、 \mathrm{Ba}-\mathrm{Ru}(1: 1) / \mathrm{Al}_{2} \mathrm{O}_{3}$ 和 $\mathrm{Ba}-\mathrm{Ru}(3: 1) /$ $\mathrm{Al}_{2} \mathrm{O}_{3}$ 催化剂中钉物种均以 $\mathrm{RuO}_{2}$ 形态存在, 这是由 于样品暴露在空气中表面被氧化的原因. 而对于 $\mathrm{Ba}-\mathrm{Ru}(5: 1) / \mathrm{Al}_{2} \mathrm{O}_{3}$ 催化剂, 其 $\mathrm{Ru} 3 d_{5 / 2}$ 比 $\mathrm{RuO}_{2}$ 低 0.4 $\mathrm{eV}$, 比 $\mathrm{Ru}^{0}$ 高 $0.2 \mathrm{eV}$, 钓物种极有可能以 $\mathrm{Ru}^{0}$ 形态存 在 ${ }^{[33]}$, 我们认为其原因可能是由于加入的 $\mathrm{BaO}$ 助剂 量较大, 大量 $\mathrm{BaO}$ 包裹在 $\mathrm{Ru}$ 粒子的表面, $\mathrm{BaO}$ 作为 电子助剂倾向于将电子传递给钓物种, 从而在一定 程度上抑制了 $\mathrm{RuO}_{2}$ 的形成. 然而, 这种大剂量的包 覆作用也同时造成 $\mathrm{BaO}-\mathrm{Ru}$ 界面难于暴露在反应气 氛中, 造成活性的下降 (表 2). 图 10(b) 为 Ba-Ru/ $\mathrm{Al}_{2} \mathrm{O}_{3}-2$ 催化剂体系的钡助剂的 XPS 谱图, Ba- $\mathrm{Ru}(1$ : 1) $/ \mathrm{Al}_{2} \mathrm{O}_{3} 、 \mathrm{Ba}-\mathrm{Ru}(3: 1) / \mathrm{Al}_{2} \mathrm{O}_{3}$ 和 $\mathrm{Ba}-\mathrm{Ru}(5: 1) / \mathrm{Al}_{2} \mathrm{O}_{3}$ 中的 $\mathrm{Ba} 3 d_{5 / 2}$ 分别位于 $780.2 、 780.4$ 和 $780.3 \mathrm{eV}$, 基本没有 变化, XRD 谱图表明 $\mathrm{Ba}-\mathrm{Ru}(1: 1) / \mathrm{Al}_{2} \mathrm{O}_{3}$ 中钡物相只 有 $\mathrm{BaCO}_{3}$, 而 $\mathrm{Ba}-\mathrm{Ru}(3: 1) / \mathrm{Al}_{2} \mathrm{O}_{3}$ 和 $\mathrm{Ba}-\mathrm{Ru}(5: 1) / \mathrm{Al}_{2} \mathrm{O}_{3}$ 中 则同时存在 $\mathrm{BaCO}_{3}$ 和 $\mathrm{BaAl}_{2} \mathrm{O}_{4}$ 物相.

\section{3 结 论}

载体的物理性质, 如结构、织构性质影响了 $\mathrm{Ru}$ 粒子在其表面的分散状态以及尺寸; 载体的化学性 质, 如表面官能团、表面酸碱性质影响了 $\mathrm{Ru}$ 物种的 还原性质和电子密度. $\mathrm{BaO}$ 助剂添加量不同导致 $\mathrm{BaO}$ 与 $\gamma-\mathrm{Al}_{2} \mathrm{O}_{3}$ 的作用力不同, $\mathrm{BaO}$ 与催化剂体系钉 物种的作用程度不同, 从而进一步影响催化剂的氨 合成活性, 适量 $\mathrm{BaO}$ 的加入能够极大促进反应活 性, 此时, $\mathrm{BaO}$ 同时起到促进钓物种的还原以及调 节体系碱性的作用, 过量的 $\mathrm{BaO}$ 的加入则会由于这 两种作用强弱的失调而降低催化剂活性. 对于不同 来源的 $\gamma-\mathrm{Al}_{2} \mathrm{O}_{3}$, 这种最佳量也是不相同的, $3 \mathrm{MPa}$, $5000 \mathrm{~h}^{-1}, \mathrm{Ba}-\mathrm{Ru}(3: 1) / \mathrm{Al}_{2} \mathrm{O}_{3}-1$ 催化剂在 $450{ }^{\circ} \mathrm{C}$ 活性为 $210.3 \mathrm{~mL} \cdot \mathrm{g}^{-1} \cdot \mathrm{h}^{-1}$, 而相同反应条件下 $\mathrm{Ba}-\mathrm{Ru}(1: 1) /$ $\mathrm{Al}_{2} \mathrm{O}_{3}$-2 在助剂含量远小于前者情况下达到 $221 \mathrm{~mL}$ ・ $\mathrm{g}^{-1} \cdot \mathrm{h}^{-1}$.

\section{References}

1 Liang, C. H.; Wei, Z. B.; Xin, Q.; Li, C. Appl. Catal. A, 2001, 208: 193

2 Yang, X. L.; Hu, B.; Xia, C. G.; Xiong, X. M.; Mu, X. Y. Ind. Catal., 2010, 18: 5 [杨晓龙, 胡 斌, 夏春谷, 熊绪茂, 慕新元. 工业催化, 2010, 18: 5]

3 You, Z. X.; Inazu, K.; Aika, K.; Baba, T. J. Catal., 2007, 251: 321

4 Zhang, X. B.; Li, X. N.; Huo, C.; Cen, Y. Q.; Zhu, Y. F.; Liu, H. Z. Chin. J. Catal., 2002, 23: 207 [张新波, 李小年, 霍 超, 岑 亚青, 祝一峰, 刘化章. 催化学报, 2002, 23: 207]

5 Szmigiel, D.; Raróg-Pilecka, W.; MiŚkiewicz, E.; Gliński, M.; Kielak, M.; Kaszkur, Z.; Kowalczyk, Z. Appl. Catal. A, 2004, 273: 105

6 Xu, Q. C.; Lin, J. D.; Fu, X. Z.; Liao, D. W. Catal. Commun., 2008, 9: 1214 
7 Ni, J.; Wang, R.; Lin, J. X.; Wei, K. M. Acta Phys. -Chim. Sin., 2009, 25(3): 519 [倪 军, 王 榕, 林建新, 魏可镁. 物理化学 学报, 2009, 25(3): 519]

8 Luo, X. J.; Wang, R.; Ni, J.; Lin, J. X.; Lin, B. Y.; Xu, X. M.; Wei, K. M. Catal. Lett., 2009, 133: 382

9 Larichev, Y. V.; Moroz, B. L.; Zaikovskii, V. L.; Yunusov, S. M.; Kalyuzhnaya, E. S.; Shur, V. B.; Bukhtiyarov, V. I. J. Phys. Chem. C, 2007, 111: 9427

10 Miyazaki, A.; Balint, I.; Aika, K.; Nakano, Y. J. Catal., 2001, 204: 364

11 Kadowaki, Y.; Aika, K. J. Catal., 1996, 161: 178

12 Siporin, S. E.; Davis, R. J. J. Catal., 2004, 225: 359

13 Wang, R.; Wei, K. M.; Lin, J. X.; Yu, X. J.; Mao, S. L. Ind. Catal., 2005, 13: 31 [王 榕, 魏可镁, 林建新, 俞秀金, 毛树禄. 工业 催化, 2005, 13: 31]

14 Mao, S. L.; Wang, R.; Yu, X. J.; Lin, J. X.; Wei, K. M. Chin. Rare Earth, 2004, 25: 18 [毛树禄, 王 榕, 俞秀金, 林建新, 魏可镁. 稀土, 2004, 25: 18]

$15 \mathrm{Fu}, \mathrm{W}$. J. The Preparation and study of the ruthenium catalysts for ammonia synthesis [D]. Fuzhou: Fuzhou University, 2003 [傅武 俊. 钉催化剂的制备及氨合成催化性能的研究[D]. 福州: 福州 大学, 2003]

16 Liu, H. Z. Ammoina synthesis catalysis: practice and theory. Beijing: Chemical Industry Press, 2007: 309, 293 [刘化章. 氨合 成催化剂实践与理论. 北京: 化学工业出版社, 2007: 293, 309]

17 Okal, J. Catal. Commun., 2010, 11: 508

18 Huo, C.; Yan, G.; Zheng, Y. F.; Yu, F. W.; Liu, H. Z. Chin. J. Catal., 2007, 28: 484 [霍 超, 晏 刚, 郑遗凡, 于凤文, 刘化 章. 催化学报, 2007, 28: 484]
19 Sharma, L. D.; Kumar, M.; Saxena, A. K.; Chand, M.; Gupta, J. K. J. Mol. Catal. A, 2002, 185: 135

20 Li,Y.; Yang, Q. H.; Yang, J.; Li, C. Microporous Mesoporous Mat., 2006, 91: 85

21 Yuan, Z. Y.; Wang, J. Z.; Zhang, Z. L.; Chen, T. H.; Li, H. X. Microporous Mesoporous Mat., 2001, 43: 227

22 Groen, J. C.; Pérez-Ramírez, J. Appl. Catal. A, 2004, 268: 121

23 Jacobsen, C. J. H.; Dahl, S.; Hansen, P. L.; Törnqvist, E.; Jensen, L.; Topsøe, H.; Prip, D. V.; Møenshaug, P. B.; Chorkendorff, I. J. Mol. Catal. A, 2000, 163: 19

24 Seetharamulu, P.; Siva Kumar, V.; Padmasri, A. H.; David Raju, B.; Rama Rao, K. S. J. Mol. Catal. A, 2007, 263: 253

25 Seetharamulu, P.; Hari Prasad Reddy, K.; Padmasri, A. H.; Rama Rao, K. S.; David Raju, B. Catal. Today, 2009, 141: 94

26 Huo, C.; Xia, Q. H.; Luo, Y.; Yang, X. Z.; Liu, H. Z. Chin. J. Catal., 2009, 30: 537 [霍 超, 夏庆华, 骆 燕, 杨霞珍, 刘化 章. 催化学报, 2009, 30: 537]

27 Hanse, T. W.; Wagner, J. B.; Hansen, P. L.; Dahl, S.; Topsøe, H.; Jacobsen, C. J. H. Science, 2001, 294: 1508

28 Dahl, S.; Logadottir, A.; Jacobsen, C. J. H.; Nørskov, J. K. Appl. Catal. A, 2001, 222: 19

29 Zhao, Y. Ind. Catal., 2002, 10: 54 [赵 琰, 工业催化, 2002, 10: 54

30 Walker, G. S.; Pyke, D. R.; Werrett, C. R.; Williams, E.; Bhattacharya, A. K. Appl. Surf. Sci., 1999, 147: 228

31 Somorjai, G. A. Science, 1978, 201: 489

32 Nagai, M.; Koizumi, K.; Omi, S. Catal. Today, 1997, 35: 393

33 Elmasides, C.; Kondarides, D. I., Grünert, W.; Verykios, X. E. J. Phys. Chem. B, 1999, 103: 5227 